Der folgende Artikel, den wir im Rahmen einer Medienpartnerschaft zwischen dem Schweizerischen Ärzteverlag EMH und den Organisatoren des 7. Schweizerischen eHealthcare Kongresses in Nottwil publizieren, wurde der SÄZ von den
Kongressverantwortlichen in der vorliegenden Form zugestellt. Die Redaktion nimmt bei Beiträgen, die Bestandteil einer Medienpartnerschaft sind, keinen Einfluss auf Themenwahl und Autor(en).

Die Redaktion

\title{
Mikrokosmos E-Health: Und es gibt ihn schon!
}

Marc Oertle

Korrespondenz:

Dr. med. Marc Oertle

Spital STS AG, Spital Thun

Krankenhausstrasse 12

CH-3600 Thun

marc.oertle@spitalthun.ch
Seit einigen Wochen ist die nationale Strategie E-Health vom Bundesrat abgesegnet. Ambitiös in der Zielsetzung, soll sie vorab den Spitälern, aber auch den Arztpraxen den Weg in die nächsten knapp zehn Jahre weisen. Während Exponenten und Politiker darüber streiten, ob der Fahrplan, die Stossrichtung und die Massnahmen die richtigen sind, ob es Sinn ergibt, eine E-Health-Strategie zu haben, wenn keine eigentliche Health-Strategie auszumachen ist, und wieweit nationale Vorgaben kantonal umgesetzt werden sollen, blühen in der Schweiz an vielen Orten schon schöne Ergebnisse von E-Health.

Am Beispiel der Spital Simmental-ThunSaanenland AG soll aufgezeigt werden, was mit einer gezielten Strategie und konsequenten Umsetzung schon heute erreicht werden kann und somit den Weg zur «nahtlos integrierten und verfügbaren» Patientenakte vorbereiten hilft.

\section{Interdisziplinäre Patientenakte}

Was vor sieben Jahren mit einer vorab ärztlichen Dokumentation begonnen hat, ist aktuell zur interdisziplinären, mittels Wireless-LAN ubiquitär, d.h. vor allem bettseitig, verfügbaren Patientenakte geworden. Sowohl die Dokumentation der Ärzte wie auch jene der Pflege sind vollständig elektronisch abgebildet. Viele paramedizinische Dienste sind ebenso in das System eingebunden wie z.B. die Radiologie. Anmeldungen geschehen elektronisch und computerunterstützt, eine Information zur Erkennung einer Nierenfunktionsstörung beim Planen eines CT-Termins ist dabei selbstverständlich. Doppelt geführte Dokumentationen gehören der Vergangenheit an, alle Berufsgruppen profitieren gegenseitig von auf ihre Bedürfnisse zugeschnittenen Informationen. Wo immer möglich, werden Daten automatisiert generiert, so z.B. die Leistungs- und Materialerfassung bei der strukturierten Anmeldung einer Röntgenuntersuchung.

\section{Regionales Zentrum und Teleradiologie}

Die Spital STS AG weist insgesamt vier Standorte auf. Die gesamte klinische Dokumentation wird dabei zentral verwaltet. Die Falldokumentation wird bei einer Verlegung nahtlos weitergeführt. Darüber hinaus werden die «Aussen»-Standorte via Teleradiologie durch das Radiologenteam am Standort Thun geführt. Beides, Technologie- und Personalzentralisierung, führt dabei zu effizienteren und kostensparenden Abläufen.

\section{Qualitäts- und Prozessoptimierung}

Bereits seit fünf Jahren im Produktivbetrieb ist die elektronische ärztliche Verordnung von Medikamenten. Diese schliesst Interaktionschecks, Allergiewarnungen und nierenfunktionsbasierte Anpassungen mit ein. Zusammen mit den damit entstehenden Qualitätsverbesserungen steht darüber hinaus ein durch die Verordnung gesteuertes Medikamentenbereitstellungssystem («elektronischer Medikamentenschrank») zur Verfügung, das weitere Qualitätsverbesserungen bringt. Der ganze Medikationsprozess bis zum Austrittsrezept und zur Validierung durch die Spitalapotheke wird elektronisch kontrolliert und optimiert. Auch über 130 Chemotherapieschemata werden elektronisch kontrolliert verordnet und ebenfalls an ein spezialisiertes Programm weitergeleitet, das die Zubereitung der Zytostatika leitet.

Aber auch andere Bereiche profitieren von der integralen Abbildung: So können im Klinikinformationssystem verordnete Labor- oder Röntgenuntersuchungen jederzeit eingesehen und wenn möglich nachverordnet oder für Terminabfragen kontrolliert werden. Der Patientenprozess wird so immer mehr zu einer zentral steuerbaren Einheit.

Mit der unlängst eingeführten semantischen Interpretation sind wir zudem in der Lage, RealTime-ICD-10-Codes automatisch aus der aktuellen Problemliste zu generieren. Als Spital, das seit mehreren Jahren mittels DRG abrechnet, ergibt sich dadurch die Möglichkeit, den $\mathrm{Pa}-$ 
tientenprozess DRG-zielgerichtet zu steuern, solange der Patient noch im Spital ist!

\section{Patientensicherheit mit RFID}

Neben der Sicherheit im Medikationsprozess bietet das jüngste Kind der Entwicklung eine markante Steigerung der Patientensicherheit. Die mittels eines Chiparmbands identifizierten Patienten erhalten jederzeit z.B. Bluttransfusionen nur, wenn das Identifikationssystem sie freigibt. Da von der Verordnung zur Blutprobe bis hin zur Bluttransfusion alle Teile der Prozesskette medienbruchfrei abgebildet sind, gehören Verwechslungen und Falschapplikationen der Vergangenheit an. Allerdings erfordern diese zusätzlichen Sicherheitsmassnahmen auch zusätzliche Arbeitsschritte. Durch ein generisches Konzept der Architektur werden nach und nach weitere Elemente wie die Medikation oder Implantate direkt über das Armband abgeglichen.

\section{Interoperabilität und Standardisierung}

Durch konsequente Verwendung von Standards gelingt es, zahlreiche Systeme mit einem minimalen Aufwand derart zu verketten, dass Informationen zeitgerecht und ohne Zusatzaufwand zwischen den Programmen ausgetauscht werden können. Dabei werden Entlastungen für das Personal möglich, da bisherige Arbeitsschritte vereinfacht oder gar vollständig abgeschafft werden können. Informationen sind am richtigen Ort verfügbar und in einer Art präsentiert, die eine integrale Arbeitsweise zulässt. Selbstverständlich kann dabei jederzeit auf das elektronische Archiv zurückgegriffen werden, dass teilweise über 15 Jahre zurückreicht.

\section{Data Warehouse:}

\section{um zu wissen, was wir $\mathbf{t}(\mathbf{h})$ un}

Eine Data-Warehouse-Lösung ist oft reserviert, um administrative Kennzahlen zu analysieren. Obwohl wesentlich komplexer im Aufbau, ist der Einbezug klinischer Daten um so aufschlussreicher in der Aussage. Dank der Einbindung der kompletten Medikationsverordnung können DRG- oder diagnosegerichtete Analysen über das Verordnungsspektrum gemacht wer- den. Das kann dazu dienen, Überverordnungen zu erkennen, die Ärzteschaft zielgerichtet zu informieren und damit nicht nur den Medikationsverbrauch zu optimieren, sondern allenfalls auch positiven Einfluss auf die Kosten und z.B. die Resistenzlage bestimmter Medikamente zu nehmen.

\section{Vom Mikro- zum Makrokosmos}

Man mag argumentieren, dass solch regionale Lösungen zwar begrüssenswert sind, zur nationalen Strategie E-Health aber wenig beitragen. Das ist ein Irrtum. Es gibt in der Schweiz aktuell zahlreiche regionale und kantonale Lösungen und Projekte, die institutsintern oder bereits mit Einbezug der Hausärzte oder weiterer ambulanter Leistungserbringer weit fortgeschritten sind. Wenn sich - wie dies in unserem Fall Tatsache ist - die Realisierungen an internationalen Standards (vorab, aber nicht abschliessend, EAN, EPC, HL-7, XML, DICOM, IHE) und nicht an proprietären Lösungen orientieren, dann besteht Gewähr, dass der zukünftige interkantonale und nationale Datenaustausch in realistischem Zeitraum konstruiert werden kann. Analog standardisierten Legobauteilen können so in den nächsten zehn Jahren die regional gelösten Hausaufgaben zum nationalen Haus zusammengebaut werden - vorausgesetzt, das nationale Haus verhält sich auch standardkonform. «E-Health im Wunderland» könnte so $\mathrm{zu}$ «E-Health im Legoland» und damit real werden. Zudem gilt es, aus diesen regionalen Lösungen grösstmöglichen Profit im Sinne der Lehren $\mathrm{zu}$ ziehen und diese Mikrokosmoserfahrungen in den nächsten Jahren in den Makrokosmos einfliessen zu lassen.

7. Schweizerischer eHealthcare Kongress Konferenz \& Fachausstellung

Mittwoch/Donnerstag, 26./27. September 2007 GZI Seminar- und Kongresshotel, Nottwil LU

Das Konferenzprogramm und weitere Informationen finden sich unter www.ehealthcare.ch. 